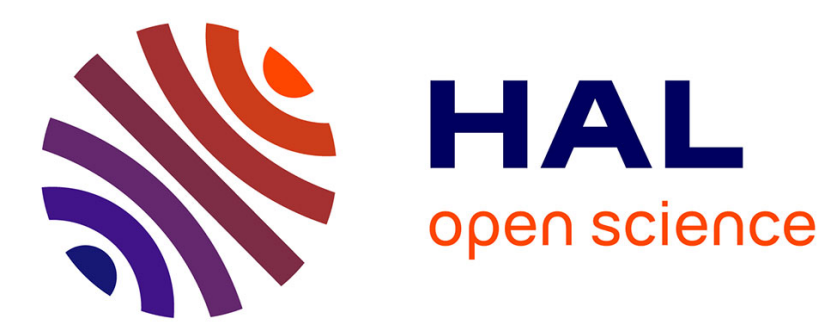

\title{
A functional limit theorem for $\eta$-weakly dependent processes and its applications
}

\author{
Jean-Marc Bardet, Paul Doukhan, José Rafael León
}

\section{To cite this version:}

Jean-Marc Bardet, Paul Doukhan, José Rafael León. A functional limit theorem for $\eta$-weakly dependent processes and its applications. Statistical Inference for Stochastic Processes, 2008, 11 (3), pp.265-280. 10.1007/s11203-007-9015-y . hal-00126530

\section{HAL Id: hal-00126530 \\ https://hal.science/hal-00126530}

Submitted on 25 Jan 2007

HAL is a multi-disciplinary open access archive for the deposit and dissemination of scientific research documents, whether they are published or not. The documents may come from teaching and research institutions in France or abroad, or from public or private research centers.
L'archive ouverte pluridisciplinaire HAL, est destinée au dépôt et à la diffusion de documents scientifiques de niveau recherche, publiés ou non, émanant des établissements d'enseignement et de recherche français ou étrangers, des laboratoires publics ou privés. 


\title{
A functional limit theorem for $\eta$-weakly dependent processes and its applications
}

\author{
Jean-Marc Bardet ${ }^{a}$, Paul Doukhan ${ }^{b}$, José Rafael León ${ }^{c \dagger}$ \\ $a \ddagger$ SAMOS-MATISSE, Université Paris I, 90 rue de Tolbiac, 75013 Paris, FRANCE, bardet@univ-paris1.fr \\ ${ }^{b}$ LS-CREST, Timbre J340, 3 avenue Pierre Larousse, 92240 Malakoff, FRANCE and SAMOS-MATISSE, \\ Université Paris I, 90 rue de Tolbiac, 75013 Paris, FRANCE, doukhan@ensae.fr \\ ${ }^{c}$ Universidad Central de Venezuela, Escuela de Matemática., Ap. 47197 Los Chaguaramos, Caracas 1041-A \\ VENEZUELA, jleon@euler.ciens.ucv.ve
}

\begin{abstract}
We prove a general functional central limit theorem for weak dependent time series. A very large variety of models, for instance, causal or non causal linear, $\operatorname{ARCH}(\infty)$, bilinear, Volterra processes, satisfies this theorem. Moreover, it provides numerous application as well for bounding the distance between the empirical mean and the Gaussian measure than for obtaining central limit theorem for sample moments and cumulants.
\end{abstract}

Keywords: Central limit theorem, Weakly dependent processes, Sample moments and cumulants

\section{Introduction}

In this paper, we consider the following empirical mean,

$$
S_{n}=\frac{1}{\sqrt{n}} \sum_{k=1}^{n} h\left(x_{k}\right)=\frac{1}{\sqrt{n}} \sum_{k=1}^{n} Y_{k}
$$

where $h: \mathbb{R}^{d} \rightarrow \mathbb{R}$ is a function and $\left(x_{n}\right)_{n \in \mathbb{Z}}$ with values in $\mathbb{R}^{d}$ is a stationary zero mean sequence that satisfying certain conditions. We study the case (see the conditions below) where $S_{n}$ converges in law to a Gaussian distribution. More precisely, the aim of this paper will be to specify conditions to obtain a decay rate to 0 of $\left|\Delta_{n}(\phi)\right|$ with

$$
\Delta_{n}(\phi)=\mathbb{E}\left(\phi\left(S_{n}\right)-\phi(N)\right),
$$

for $\phi$ a $\mathcal{C}^{3}(\mathbb{R})$ function with bounded derivatives up to order 3 , where $N$ is a Gaussian random variable satisfying $N \sim \mathcal{N}\left(0, \sigma^{2}\right)$ with $\sigma^{2}=\sum_{k \in \mathbb{Z}} \operatorname{Cov}\left(h\left(x_{0}\right), h\left(x_{k}\right)\right)$.

Let us precise now the different assumptions for the times series and functions considered in (1). First, we have chosen to work in the frame of $\eta$-weakly dependent processes. This very general class of dependent processes was introduced in the seminal paper of Doukhan and Louhichi (1999) to generalize and avoid certain difficulties linked of strong mixing property. Indeed, this frame of dependence includes a lot of models like causal or non causal linear, bilinear, strong mixing processes or also dynamical systems. Secondly, this property of dependence is independent of the marginal distribution of the time series, that can be as well a discrete one, Lebesgue measurable one or else. Thirdly, non causal processes can be as well studied as causal ones with this property of dependence, in contrary of strong mixing processes or martingales. Finally, these definitions of dependence can be more easily proved and used in a lot of statistic contexts, in particular in the case of functions of the time series, than the strong mixing one.

$\dagger$ This author aknowledges the program ECOS-NORD of Fonacit, Venezuela, for its support.

¥ Author for correspondence.

(c) 2006 Kluwer Academic Publishers. Printed in the Netherlands. 
The definition of such $\eta$-weak dependent property is the following. A process $X=\left(X_{n}\right)_{n \in \mathbb{Z}}$ with values in $\mathbb{R}^{d}$ is a so-called $\eta$-weakly dependent process when it exists a sequence $\left(\eta_{r}\right)_{r \in \mathbb{N}}$ converging to 0 satisfying:

$$
\left|\operatorname{Cov}\left(g_{1}\left(X_{i_{1}}, \ldots, X_{i_{u}}\right), g_{2}\left(X_{j_{1}}, \ldots, X_{j_{v}}\right)\right)\right| \leq\left(u \cdot\left(\operatorname{Lip} g_{1}\right) \cdot\left\|g_{2}\right\|_{\infty}+v \cdot\left(\operatorname{Lip} g_{2}\right) \cdot\left\|g_{1}\right\|_{\infty}\right) \cdot \eta_{r}
$$

for all $\left\{\begin{array}{l}\bullet(u, v) \in \mathbb{N}^{*} \times \mathbb{N}^{*} ; \\ \bullet\left(i_{1}, \ldots, i_{u}\right) \in \mathbb{Z}^{u} \text { and }\left(j_{1}, \ldots, j_{v}\right) \in \mathbb{Z}^{v} \text { with } i_{1} \leq \cdots \leq i_{u}<i_{u}+r \leq j_{1} \leq \cdots \leq j_{v} ; \\ \bullet \text { functions } g_{1}: \mathbb{R}^{u d} \rightarrow \mathbb{R} \text { and } g_{2}: \mathbb{R}^{v d} \rightarrow \mathbb{R} \text { satisfying } \\ \left\|g_{1}\right\|_{\infty} \leq \infty,\left\|g_{2}\right\|_{\infty} \leq \infty, \text { Lip } g_{1}<\infty \text { and Lip } g_{2}<\infty .\end{array}\right.$

A lot of usual time series are $\eta$-weakly dependent. Different examples of such time series will be studied in the following section: strong mixing processes (see Doukhan and Louhichi, 1999), $\operatorname{GARCH}(p, q)$ or $\operatorname{ARCH}(\infty)$ processes (see Doukhan et al., 2004), causal or non causal linear processes (see Doukhan and Lang, 2002), causal or non causal bilinear processes (see Doukhan et al., 2005) and causal or non causal Volterra processes (see Doukhan, 2003). Now, we can specify the different assumptions used in the general functional central limit theorem:

Assumptions A on the sequence $\left(x_{n}\right)_{n}$ :

(in the sequel, the norm is $\left|\left(u_{1}, \ldots, u_{d}\right)\right|=\max \left\{\left|u_{1}\right|, \ldots,\left|u_{d}\right|\right\}$ for $\left(u_{1}, \ldots, u_{d}\right) \in \mathbb{R}^{d}$ )

1. there exists $m$-th order moments for $\left(x_{n}\right)_{n}$ with $m>2$;

2. $\left(x_{n}\right)_{n \in \mathbb{Z}}$ is a $\eta$-weakly dependent process (defined from the inequality (3)) with values in $\mathbb{R}^{d}$, and the sequence $\eta=\left(\eta_{r}\right)_{r \in \mathbb{N}}$ satisfies:

$$
0<\eta_{r}=\mathcal{O}\left(r^{-\alpha}\right) \quad \text { for all } r \in \mathbb{N} \quad \text { with } \quad \alpha>0 .
$$

\section{Assumptions $\mathbf{H}$ on the function $h$ :}

1. $\mathbb{E}\left(h\left(x_{0}\right)\right)=0$;

2. There exists $a \geq 1$ and $A=A(d) \geq 1$ such that for all $u, v \in \mathbb{R}^{d}$,

$$
\begin{cases}|h(u)| & \leq A\left(|u|^{a} \vee 1\right) \\ |h(u)-h(v)| & \left.\leq A\left(|u|^{a-1}+|v|^{a-1}\right) \vee 1\right)|u-v|\end{cases}
$$

Using a Bernstein's blocks technique, we prove here the following theorem:

THEOREM 1. Let $h$ and $\left(x_{n}\right)_{n \in \mathbb{Z}}$ satisfy respectively assumptions $H$ and $A$, with $m>2 a$. If $\alpha>\max \left(3 ; \frac{2 m-1}{m-2 a}\right)$, then $\sigma^{2}=\sum_{k=-\infty}^{\infty} \operatorname{Cov}\left(h\left(x_{0}\right), h\left(x_{k}\right)\right)<\infty$, and for any $Z \sim \mathcal{N}(0,1)$ random variable, for any $\phi \in \mathcal{C}^{3}(\mathbb{R})$ with bounded derivatives, there exists $c>0$ such that:

$$
\left|\mathbb{E}\left(\phi\left(S_{n}\right)-\phi(\sigma \cdot Z)\right)\right| \leq c \cdot n^{-\lambda} \quad \text { with } \quad \lambda=\frac{\alpha(m-2 a)-2 m+1}{2(m+a-1+\alpha \cdot m)} .
$$


Even if this result may be not optimal in term of the conditions linking the moment assumption with the decay rate of weak dependence of the time series, this however concerns a lot of models and open new perspectives of treatments for non causal processes. Moreover, if $\alpha \rightarrow \infty$ and $m \rightarrow \infty$ are large enough, then $\lambda \rightarrow \frac{1}{2}$. In such an asymptotic case, the rate of convergence $n^{-\lambda}$ is close to the rate of sequence of i.i.d. random variables (that is $1 / \sqrt{n}$ ).

The remainder of this paper is organized as follows. The following Section 2 is devoted to statistical applications for different examples of $\eta$-weakly time series. Section 3 contains the main proof of the general functional central limit theorem.

\section{Applications of the functional central limit theorem}

\subsection{Time series satisfying the Assumptions A}

\section{Causal GARCH and ARCH( $(\infty)$ processes}

The famous and from now on classical $\operatorname{GARCH}\left(q^{\prime}, q\right)$ model was introduced by Engle (1982) and Bollerslev (1986) and is given by relations

$$
X_{k}=\rho_{k} \cdot \xi_{k} \quad \text { with } \rho_{k}^{2}=a_{0}+\sum_{j=1}^{q} a_{j} X_{k-j}^{2}+\sum_{j=1}^{q^{\prime}} c_{j} \rho_{k-j}^{2},
$$

where $\left(q^{\prime}, q\right) \in \mathbb{N}^{2}, a_{0}>0, a_{j} \geq 0$ and $c_{j} \geq 0$ for $j \in \mathbb{N}$ and $\left(\xi_{k}\right)_{k \in \mathbb{Z}}$ are i.i.d. random variables with zero mean (for an excellent survey about ARCH modelling, see Giraitis et al., 2005). Under some additional conditions, the GARCH model can be written as a particular case of $\mathrm{ARCH}(\infty)$ model (introduced in Robinson, 1991) that satisfied:

$$
X_{k}=\rho_{k} \cdot \xi_{k} \quad \text { with } \rho_{k}^{2}=b_{0}+\sum_{j=1}^{\infty} b_{j} X_{k-j}^{2}
$$

with a sequence $\left(b_{j}\right)_{j}$ depending on the family $\left(a_{j}\right)$ and $\left(c_{j}\right)$ in the case of $\operatorname{GARCH}\left(q^{\prime}, q\right)$ process. Then,

PROPOSITION 1. [See Doukhan et al, 2005] Let h satisfies assumption H. Let $X$ be a stationary $A R C H(\infty)$ time series following equation (7), such that it exists $m>2 a$ satisfying $\mathbb{E}\left(\left|\xi_{0}\right|^{m}\right)<\infty$, with the condition of stationarity, $\left\|\xi_{0}\right\|_{m}^{2} \cdot \sum_{j=1}^{\infty}\left|b_{j}\right|<1$. Then, if:

- it exists $C>0$ and $\mu \in] 0,1\left[\right.$ such that $\forall j \in \mathbb{N}, 0 \leq b_{j} \leq C \cdot \mu^{-j}$, then $X$ is a $\eta$-weakly dependent process with $\eta_{r}=\mathcal{O}\left(e^{-c \sqrt{r}}\right)$ and $c>0$ (this is the case of $\operatorname{GARCH}\left(q^{\prime}, q\right)$ processes) and (5) is satisfied.

- it exists $C>0$ and $\nu>\max \left(4 ; \frac{3 m-2 a-1}{m-2 a}\right)$ such that $\forall j \in \mathbb{N}, 0 \leq b_{j} \leq C \cdot j^{-\nu}$, then $X$ is a $\eta$-weakly dependent process with $\theta_{r}=\mathcal{O}\left(r^{-\nu+1}\right)$ and (5) is satisfied. 


\section{Causal Bilinear processes}

Assume that $X=\left(X_{k}\right)_{k \in \mathbb{Z}}$ is a bilinear process (see the seminal paper of Giraitis and Surgailis, 2002) satisfying the equation:

$$
X_{k}=\xi_{k}\left(a_{0}+\sum_{j=1}^{\infty} a_{j} X_{k-j}\right)+c_{0}+\sum_{j=1}^{\infty} c_{j} X_{k-j} \quad \text { for } k \in \mathbb{Z},
$$

where $\left(\xi_{k}\right)_{k \in \mathbb{Z}}$ are i.i.d. random variables with zero mean and such that $\left\|\xi_{0}\right\|_{p}<+\infty$ with $p \geq 1$, and $a_{j}, c_{j}, j \in \mathbb{N}$ are real coefficients.

PROPOSITION 2. [See Doukhan et al., 2004] Let h satisfies assumption H. Let $X$ be a stationary bilinear time series satisfying equation (8) with $c_{0}=0, \mathbb{E}\left(\left|\xi_{0}\right|^{m}\right)<\infty$ and $\left\|\xi_{0}\right\|_{m} \cdot\left(\sum_{j=1}^{\infty}\left|a_{j}\right|+\right.$ $\left.\sum_{j=1}^{\infty}\left|c_{j}\right|\right)<1$ with $m>2 a$. If:

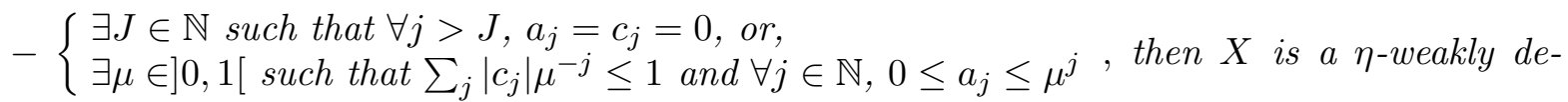
pendent process with $\eta_{r}=\mathcal{O}\left(e^{-c \sqrt{r}}\right)$, constant $c>0$ and (5) is satisfied.

- $\forall j \in \mathbb{N}, c_{j} \geq 0$, and $\exists \nu_{1}>2$ and $\exists \nu_{2}>0$ such that $a_{j}=\mathcal{O}\left(j^{-\nu_{1}}\right), \sum_{j} c_{j}<1$ and $\sum_{j} c_{j} j^{1+\nu_{2}}<\infty$, then $X$ is a $\eta$-weakly dependent process with $\theta_{r}=\mathcal{O}\left(\left(\frac{r}{\log r}\right)^{-d}\right)$, where

$d=\min \left(\nu_{1}-1 ; \frac{\nu_{2} \cdot \delta}{\delta-\nu_{2} \cdot \log 2}\right)$ and $\delta=\log \left(1+\frac{1-\sum_{j} c_{j}}{\sum_{j} c_{j} j^{1+\nu_{2}}}\right)$. Moreover, if $d>\max \left(3 ; \frac{2 m-1}{m-2 a}\right)$, then (5) is satisfied.

\section{Non-causal (two-sided) linear processes}

PROPOSITION 3. [See Doukhan and Lang, 2002, $p$. 3] Let h satisfies assumption H. Let X be a stationary bilinear time series satisfying equation

$$
X_{k}=\sum_{j=-\infty}^{\infty} a_{j} \xi_{k-j} \quad \text { for } k \in \mathbb{Z}
$$

with $\left(a_{k}\right)_{k \in \mathbb{Z}} \in \mathbb{R}^{\mathbb{Z}}$ and $\left(\xi_{k}\right)_{k \in \mathbb{Z}}$ a sequence of zero mean i.i.d. random variables such that $\mathbb{E}\left(\left|\xi_{0}\right|^{m}\right)<$ $\infty$ for $m>2 a$. Assume that $a_{k}=\mathcal{O}\left(|k|^{-\mu}\right)$ with $\mu>\max \left(\frac{7}{2} ; \frac{5 m-2 a-2}{2(m-2 a)}\right)$. Then $X$ is a $\eta$-weakly dependent process with $\eta_{r}=\mathcal{O}\left(\frac{1}{r^{\mu-1 / 2}}\right)$ and (5) is satisfied.

REMARK 1. Despite the quite simplicity of this model, it exists very few results concerning the dependence of the two-sided linear processes. The main reason of this is difficulty to use martingale or mixing properties for a non-causal process. However, in Rosenblatt (2000, p. 52) a non-efficient strong mixing property for two-sided linear processes was given, but under restrictive conditions and with. The case of strongly dependent two-sided linear processes was also treated by Giraitis and Surgailis (1990) or Horvath and Shao (1999) but only with $a_{k}=\mathcal{O}\left(|k|^{-a}\right)$ for a fixed $-1<a<0$. 


\section{Non-causal Volterra processes}

Let $X=\left(X_{t}\right)_{t \in \mathbb{Z}}$ be the zero mean non causal (two-sided) and nonlinear time series, so called a non-causal Volterra process, such that for $t \in \mathbb{Z}$ :

$$
X_{k}=\sum_{p=1}^{\infty} Y_{k}^{(p)}, \quad \text { with } \quad Y_{k}^{(p)}=\sum_{\substack{j_{1}<j_{2}<\ldots<j_{p} \\ j_{1}, \ldots, j_{p} \in \mathbb{Z}}} a_{j_{1}, \ldots, j_{p}} \xi_{k-j_{1}} \cdots \xi_{k-j_{p}},
$$

where $\left(a_{j_{1}, \ldots, j_{p}}\right) \in \mathbb{R}$ for $p \in \mathbb{N}^{*}$ and $\left(j_{1}, \ldots, j_{p}\right) \in \mathbb{Z}^{p}$, and $\left(\xi_{k}\right)_{k \in \mathbb{Z}}$ a sequence of zero mean i.i.d. random variables such that $\mathbb{E}\left(\xi_{0}^{2}\right)=\sigma^{2}<\infty$ and $\mathbb{E}\left(\left|\xi_{0}\right|^{m}\right)<\infty$ with $m>0$. Such a Volterra process is a natural extension of the previous case of non-causal linear process.

PROPOSITION 4. [See Doukhan, 2003] Let h satisfies assumption H. Let $X$ be a stationary non-causal Volterra process, satisfying equation (10) with $\mathbb{E}\left(\left|\xi_{0}\right|^{m}\right)<\infty$ and

$$
\sum_{p=0}^{\infty} \sum_{\substack{j_{1}<j_{2}<\ldots<j_{p} \\ j_{1}, \ldots, j_{p} \in \mathbb{Z}}}\left|a_{j_{1}, \ldots, j_{p}}\right|^{m}\left\|\xi_{0}\right\|_{m}^{p}<\infty .
$$

Assume that the process is in some finite order chaos (there exists $p_{0} \in \mathbb{N}$ such that $a_{j_{1}, \ldots, j_{p}}=0$ for $\left.p>p_{0}\right)$ and $a_{j_{1}, \ldots, j_{p}}=\mathcal{O}\left(\max _{1 \leq i \leq p}\left\{\left|j_{i}\right|^{-\mu}\right\}\right)$ with $\mu>\max \left(2 ; \frac{m+2 a-1}{m-2 a}\right)$. Then $X$ is a $\eta$-weakly dependent process with $\eta_{r}=\mathcal{O}\left(\frac{1}{r^{\mu+1}}\right)$ and (5) is satisfied.

\section{$\underline{\text { Non-causal (two-sided) bilinear processes }}$}

As a natural generalization of causal bilinear process, Doukhan et al. (2005), Lemma 2.1, define $X=\left(X_{t}\right)_{t \in \mathbb{Z}}$ a zero mean nonlinear time series, so called a non-causal (two-sided) bilinear process. They proved the stationarity in $\mathbb{L}^{k}$ (for any $\left.\left.k \in\right] 0, \infty\right]$ ) of such a bilinear process $X=\left(X_{k}\right)_{k \in \mathbb{Z}}$ satisfying the equation:

$$
X_{k}=\xi_{k} \cdot\left(a_{0}+\sum_{j \in \mathbb{Z}^{*}} a_{j} X_{k-j}\right), \quad \text { for } k \in \mathbb{Z}
$$

where $\left(\xi_{k}\right)_{k \in \mathbb{Z}}$ are i.i.d. random bounded variables and $\left(a_{k}\right)_{k \in \mathbb{Z}}$ is a sequence of real numbers such that $\lambda=\left\|\xi_{0}\right\|_{\infty} \cdot \sum_{j \neq 0}\left|a_{j}\right|<1$.

PROPOSITION 5. [See Doukhan et al., 2005] Let $h$ satisfies assumption $H$. If $X$ is a stationary non causal bilinear process, i.e. a solution of (11), such that $\left\|\xi_{0}\right\|_{\infty} \cdot \sum_{j \neq 0}\left|a_{j}\right|<1$. Moreover, assume that the sequence $\left(a_{k}\right)_{k \in \mathbb{Z}}$ is such that: $a_{k}=\mathcal{O}\left(|k|^{-\mu}\right)$ with $\mu>\max \left(4 ; \frac{3 m-2 a-1}{m-2 a}\right)$. Then $X$ is a $\eta$-weakly dependent process with $\eta_{r}=\mathcal{O}\left(\frac{1}{r^{\mu-1}}\right)$ and (5) is satisfied.

\section{$\underline{\text { Non-causal linear processes with dependent innovations }}$}

Let $X=\left(X_{n}\right)_{n \in \mathbb{N}}$ be a zero mean stationary non causal (two-sided) linear time series satisfying equation (9) with a dependent innovation process. Following the results of Doukhan and Wintenberger (2005), if $\left(\xi_{n}\right)_{n \in \mathbb{Z}}$ is a $\eta$-weakly dependent process, then $X$ is an $\eta$-weakly dependent process: 
PROPOSITION 6. Let $h$ satisfies assumption $H$. Let $X$ be a linear time series satisfying (9) with $\left(a_{k}\right)_{k \in \mathbb{Z}} \in \mathbb{R}^{\mathbb{Z}}$ and $\left(\xi_{k}\right)_{k \in \mathbb{Z}}$ a $\eta^{(\xi)}$-weakly dependent process with zero mean, such that $\mathbb{E}\left(\left|\xi_{0}\right|^{m}\right)<\infty$ and $m>2 a$. Moreover, assume that $a_{k}=\mathcal{O}\left(|k|^{-\mu}\right)$ and $\eta_{r}^{(\xi)}=\mathcal{O}\left(r^{-\nu}\right)$. Then $X$ is a $\eta$-weakly dependent process with $\eta_{r}=\mathcal{O}\left(r^{-\nu \cdot \frac{(\mu-2)(m-2)}{(\mu-1)(m-1)}}\right)$ and (5) is satisfied if $\frac{(\mu-2)(m-2)}{(\mu-1)(m-1)} \cdot \nu \geq \max \left(3 ; \frac{2 m-1}{m-2 a}\right)$.

\subsection{Applications of the functional Central limit theorem}

1. The general functional theorem (1), which concerns the estimate of Dudley $\left|\mathbb{E}\left(\phi\left(S_{n}\right)-\phi(\sigma \cdot N)\right)\right|$, allows an interesting application: the majoration of a measure of the distance between $S_{N}$ and its Gaussian approximation. Indeed:

COROLLARY 1. Under the assumptions of Theorem 1, there exists some $c^{\prime}>0$ such that:

$$
\sup _{t \in R}\left|\mathbb{P}\left(S_{n} \leq t\right)-P(\sigma \cdot N \leq t)\right| \leq c^{\prime} \cdot n^{-\lambda / 4}, \quad \text { for } n \in \mathbb{N} .
$$

Proof of Corollary 1. Arguing as in Doukhan (1994) we consider, in expression (2), a smooth approximation $\phi_{\varepsilon, x}$ of the indicator function $\phi_{x}(t)=\mathbb{I}_{\{t \leq x\}}$; this is possible to assume that $\phi_{x} \leq \phi_{\varepsilon, x} \leq \phi_{x+\varepsilon}$ and $\left\|\phi_{\varepsilon, x}^{(j)}\right\|_{\infty} \leq C \varepsilon^{-j}$ for some constant $C$ and for $j=1,2$ or 3 . Then the result may be specified and the bound may also be written with a constant $c=C$. $\left(\left\|\phi^{\prime}\right\|_{\infty}+\left\|\phi^{\prime \prime}\right\|_{\infty}+\left\|\phi^{\prime \prime \prime}\right\|_{\infty}\right)$ for some $C$ which does not depend on $\phi$. With the notation (2), we obtain the bound: $\Delta_{n}\left(\phi_{\varepsilon, x}\right) \leq C \varepsilon^{-3} n^{-\lambda}$ for some constant (still denoted) $C>0$ and each $x \in \mathbb{R}, \varepsilon \in] 0,1]$, and $n>0$. Using the relation $\sup _{u \in \mathbb{R}} \mathbb{P}(\sigma \cdot N \in[u, u+\varepsilon)) \leq \frac{\varepsilon}{\sigma \sqrt{2 \pi}}$, the above mentioned expression is then bounded above for a suitable constant $c$ by $c\left(\varepsilon^{-3} n^{-\lambda}+\varepsilon\right)$; the choice $\varepsilon=n^{-\lambda / 4}$ thus yields the result.

REMARK 2. Unfortunately, this rate is far from being optimal as stressed by Rio (2000) which obtains rate $n^{-\rho}$ for some $\rho<1 / 3$ in the case of strongly mixing sequences. Here, in the best cases $(\lambda \rightarrow 1 / 2$ when $\alpha \rightarrow \infty$, that is for instance the case of $\operatorname{GARCH}(p, q))$, we obtain the rate $n^{-\tau}$ for some $\tau<1 / 8$.

2. The general functional theorem (1) could be applied for providing central limit theorems for sample moments or cumulants. Here, we consider a real valued time series $\left(X_{n}\right)_{n \in \mathbb{Z}}$ satisfying assumption A (with parameters $a$ and $m$ that will be specified above). Indeed, for $k \in \mathbb{N}^{*}$, denote $p=\left(p_{1}, \ldots, p_{k}\right) \in \mathbb{N}^{k},|p|=p_{1}+\cdots+p_{k}$ and assuming that $\mathbb{E}\left(X_{1}^{|p|}\right)<\infty$, define:

$$
\begin{aligned}
& m^{(p)}=\mathbb{E}\left(X_{1}^{p_{1}} X_{2}^{p_{2}} \cdots X_{k}^{p_{k}}\right), \quad \text { the moment of order } p \\
& \widehat{m}_{n}^{(p)}=\frac{1}{n} \sum_{i=1}^{n} X_{i}^{p_{1}} X_{i+1}^{p_{2}} \cdots X_{i+k-1}^{p_{k}}, \quad \text { the sample moment of order } p
\end{aligned}
$$

Then, the following results generalize the usual central limit theorems for strong mixing processes (for instance, see a survey in Rosenblatt, 1985): 
COROLLARY 2. 1. Set $k \in \mathbb{N}^{*}$ and $p=\left(p_{1}, \ldots, p_{k}\right) \in \mathbb{N}^{k},|p|=p_{1}+\cdots+p_{k}$. Let $\left(X_{n}\right)_{n \in \mathbb{Z}}$ be a real valued time series satisfying assumption $A$ with $m>2|p|$ and $\alpha>\max \left(3 ; \frac{2 m-1}{m-2|p|}\right)$. Then:

$$
\sqrt{n}\left(\widehat{m}_{n}^{(p)}-m^{(p)}\right) \underset{n \rightarrow \infty}{\stackrel{\mathcal{D}}{\longrightarrow}} \mathcal{N}\left(0, \sigma^{2}(p)\right)
$$

with $\sigma^{2}(p)=\sum_{\ell \in \mathbb{Z}}\left(\mathbb{E}\left(X_{1}^{p_{1}} X_{2}^{p_{2}} \cdots X_{k}^{p_{k}} X_{\ell+1}^{p_{1}} \cdots X_{\ell+k}^{p_{k}}\right)-\left(m^{(p)}\right)^{2}\right)$.

2. More generally, let $I \in \mathbb{N}^{*},\left(k_{1}, \ldots, k_{I}\right) \in\left(\mathbb{N}^{*}\right)^{I}$, and for $i=1, \ldots, I, p^{(i)}=\left(p_{1}^{(i)}, \cdots, p_{k_{i}}^{(i)}\right)$. Let $\left(X_{n}\right)_{n \in \mathbb{Z}}$ be a real valued time series satisfying assumption $A$ with $m>2 \max \left(\left|p_{1}\right|, \ldots,\left|p_{I}\right|\right)$ and $\alpha>\max \left(3 ; \frac{2 m-1}{m-2 \max \left(\left|p_{1}\right|, \ldots,\left|p_{I}\right|\right)}\right)$. Then:

$$
\sqrt{n}\left(\widehat{m}_{n}^{\left(p_{i}\right)}-m^{\left(p_{i}\right)}\right)_{1 \leq i \leq I} \underset{n \rightarrow \infty}{\stackrel{\mathcal{D}}{\longrightarrow}} \mathcal{N}_{I}\left(0, \Sigma\left(p_{1}, \ldots, p_{I}\right)\right),
$$

with $\Sigma\left(p_{1}, \ldots, p_{I}\right)=\left(\sum_{\ell \in \mathbb{Z}}\left(\mathbb{E}\left(X_{1}^{p_{1}^{(i)}} X_{2}^{p_{2}^{(i)}} \cdots X_{k}^{p_{k}^{(i)}} X_{\ell+1}^{p_{1}^{(j)}} \cdots X_{\ell+k}^{p_{k}^{(j)}}\right)-m^{\left(p_{i}\right)} m^{\left(p_{j}\right)}\right)\right)_{1 \leq i, j \leq I}$.

Proof. 1. Let $x_{i}=\left(X_{i}, \ldots, X_{i+k-1}\right)$ and $h^{(p)}:\left(z_{1}, \ldots, z_{k}\right) \in \mathbb{R}^{k} \mapsto z_{1}^{p_{1}} z_{2}^{p_{2}} \cdots z_{k}^{p_{k}}-m^{(p)}$ that satisfies Assumption H with $a=|p|$. Indeed, it is possible to prove that $|h(u)| \leq 2\left(m^{(p)} \vee|u|^{|p|}\right)$ and $\left.|h(u)-h(v)| \leq 2^{|p|}\left(|u|^{|p|-1}+|v|^{|p|-1}\right) \vee 1\right)|u-v|$. Then, theorem 1 can be applied.

2. Let $k=\max \left(k_{1}, \ldots, k_{I}\right)$ and $x_{i}=\left(X_{i}, \ldots, X_{i+k-1}\right)$. Let $\left(\lambda_{1}, \ldots, \lambda_{I}\right) \in \mathbb{R}^{I}$ and $h=\sum_{i=1}^{I} \lambda_{i}$. $h^{\left(p_{i}\right)}$. It is clear that $h$ satisfies Assumption $\mathrm{H}$ with $a=\max \left(\left|p_{1}\right|, \ldots,\left|p_{I}\right|\right)$ (indeed, if each $h^{\left(p_{i}\right)}$ satisfies Assumption $\mathrm{H}$ with coefficient $a_{i}=\left|p_{i}\right|$, then a linear combination of $h^{\left(p_{i}\right)}$ satisfies Assumption $\mathrm{H}$ with coefficient $\left.a=\max \left(\left|a_{1}\right|, \ldots, a_{I}\right)\right)$. Then Theorem 1 implies that $\sum_{i=1}^{I} \lambda_{i}$. $\widehat{m}_{n}^{\left(p_{i}\right)}$ satisfies a central limit theorem. It implies the multidimensional central limit theorem.

A natural and interesting example that generalizes usual central limit theorem under strong mixing conditions is the following:

EXAMPLE 1. Let $\left(X_{n}\right)_{n \in \mathbb{Z}}$ be a real valued time series satisfying assumption A. For all $m \in$ $\mathbb{N}^{*}, \ell_{1}<\cdots<\ell_{m} \in \mathbb{N}^{m}$, define $R\left(\ell_{i}\right)=\mathbb{E}\left(X_{0} X_{\ell_{i}}\right)$ and $\widehat{R}_{n}\left(\ell_{i}\right)=\frac{1}{n} \sum_{j=1}^{n} X_{j} X_{j+\ell_{i}}$. Then

$$
\sqrt{n}\left(\widehat{R}_{n}\left(\ell_{i}\right)-R\left(\ell_{i}\right)\right)_{1 \leq i \leq m} \underset{n \rightarrow \infty}{\stackrel{\mathcal{D}}{\longrightarrow}} \mathcal{N}_{k}\left(0, \Sigma\left(\ell_{1}, \ldots, \ell_{m}\right)\right), \quad \text { if } \alpha>\max \left(3 ; \frac{2 m-1}{m-4}\right)
$$

with $\Sigma\left(\ell_{1}, \ldots, \ell_{m}\right)=\left(\sum_{k \in \mathbb{Z}}\left(\mathbb{E}\left(X_{0} X_{\ell_{i}} X_{k} X_{k+\ell_{j}}\right)-R\left(\ell_{i}\right) R\left(\ell_{j}\right)\right)\right)_{1 \leq i, j \leq m}$.

Moreover, the sample skewness and sample Kurtosis satisfy also central limit theorems from the Delta-method applied to multidimensional theorem (12). 
More generally, another consequence of such a result is the central limit theorems satisfied by the estimation of the $k$-th cumulant of $X$ (obtained from the Taylor development of the characteristic function logarithm), defined by:

$$
\kappa^{(\mu)}=\operatorname{Cumulant}\left(X_{1}, X_{i_{2}}, \ldots, X_{i_{k}}\right), \quad \text { for } \mu=\left\{1, i_{2}, \ldots, i_{|\mu|}\right\} \subset\{1, \ldots, k\} .
$$

From Leonov and Shiryaev (1959), there is a relation between cumulants and moments, that is:

$$
\kappa^{(\mu)}=\sum_{u=1}^{k}(-1)^{u-1}(u-1) \sum_{\left(\mu_{1}, \ldots, \mu_{u}\right)} \prod_{j=1}^{u} m^{\left(\mu_{j}\right)},
$$

where $\left(\mu_{1}, \ldots, \mu_{u}\right)$ (in the sum) describe all possible partitions in $u$ subsets of the subset $\mu$. Then:

COROLLARY 3. Set $k \in \mathbb{N}^{*}, \mu=\left\{1, i_{2}, \ldots, i_{|\mu|}\right\} \subset\{1, \ldots, k\}$. Let $\left(X_{n}\right)_{n \in \mathbb{Z}}$ be a real valued time series satisfying assumption $A$ with $m>2|\mu|$ and $\alpha>\max \left(3 ; \frac{2 m-1}{m-2|\mu|}\right)$. Then it exists $\gamma^{2}(\mu)>0$ such that:

$$
\sqrt{n}\left(\widehat{\kappa}_{n}^{(\mu)}-\kappa^{(\mu)}\right) \underset{n \rightarrow \infty}{\stackrel{\mathcal{D}}{\longrightarrow}} \mathcal{N}\left(0, \gamma^{2}(\mu)\right),
$$

from the sample cumulant $\quad \widehat{\kappa}_{n}^{(\mu)}=\sum_{u=1}^{k}(-1)^{u-1}(u-1) \sum_{\left(\mu_{1}, \ldots, \mu_{u}\right)} \prod_{j=1}^{u} m_{n}^{\left(\mu_{j}\right)}$.

\section{Proof of Theorem 1}

From now on, $c>0$ denotes a constant which may vary from one line to the other.

First, define a truncation in order to be able to use the previous dependence condition and make Lindeberg technique work. For $T>0$, define $f_{T}(x)=(x \wedge T) \vee(-T)$ for $x \in \mathbb{R}$. Then Lip $f_{T}=1$, $\left\|f_{T}\right\|_{\infty}=T$. For $\left(u_{1}, \ldots, u_{d}\right) \in \mathbb{R}^{d}$, we denote

$$
F_{T}\left(u_{1}, \ldots, u_{d}\right)=\left(f_{T}\left(u_{1}\right), \ldots, f_{T}\left(u_{d}\right)\right)
$$

and

$$
Y_{i}=h\left(x_{i}\right), \quad Y_{i}^{(T)}=h\left(F_{T}\left(x_{i}\right)\right)-\mathbb{E}\left[h\left(F_{T}\left(x_{i}\right)\right)\right], \quad E_{i}^{(T)}=Y_{i}-Y_{i}^{(T)}
$$

LEMMA 1. Let $h$ and $\left(x_{n}\right)_{n \in \mathbb{Z}}$ satisfy respectively assumptions $H$ and $A$, with $m>2 a$. Then,

a) $\mathbb{E}\left[\left|E_{0}^{(T)}\right|\right] \leq c \cdot A \cdot T^{a-m}$ and $\mathbb{E}\left[\left(E_{0}^{(T)}\right)^{2}\right] \leq c \cdot A^{2} \cdot T^{2 a-m}$;

b) for all $i \in \mathbb{Z},\left|\operatorname{Cov}\left(Y_{0}^{(T)}, E_{i}^{(T)}\right)\right| \leq \mathbb{E}\left(\left|Y_{0}^{(T)}\right|,\left|E_{i}^{(T)}\right|\right) \leq c \cdot A^{2} \cdot T^{2 a-m}$;

c) for all $i \in \mathbb{Z},\left|\operatorname{Cov}\left(Y_{0}^{(T)}, Y_{i}^{(T)}\right)\right| \leq c \cdot A^{2} \cdot T^{2 a-1} \cdot \eta_{i}$. 
Proof of Lemma 1. First note that for $\gamma \geq 1$ such that $a \gamma \leq m$, from assumptions on $h$,

$$
\begin{aligned}
\mathbb{E}\left[\left|h\left(x_{0}\right)-h\left(F_{T}\left(x_{0}\right)\right)\right|^{\gamma}\right] & \leq A^{\gamma} \cdot \mathbb{E}\left[\left|\left(\left|x_{0}\right|^{a-1}+\left|F_{T}\left(x_{0}\right)\right|^{a-1}\right) \cdot\right| x_{0}-F_{T}\left(x_{0}\right)||^{\gamma}\right] \\
& \leq(2 A)^{\gamma} \cdot \mathbb{E}\left[\left|x_{0}\right|^{a \gamma} \cdot \mathbb{I}_{\left\{\left|x_{0}\right| \geq T\right\}}\right] \\
& \leq(2 A)^{\gamma} \cdot \mu \cdot T^{\gamma a-m} \text { (Markov inequality). }
\end{aligned}
$$

a) The assumptions on $h$ lead to

$$
\begin{aligned}
\mathbb{E}\left[\left|E_{0}^{(T)}\right|\right] & \leq \mathbb{E}\left[\left|h\left(F_{T}\left(x_{0}\right)\right)-h\left(x_{0}\right)\right|\right]+\mathbb{E}\left[\left|h\left(F_{T}\left(x_{0}\right)\right)\right|\right] \\
& \leq 2 \mathbb{E}\left[\left|h\left(F_{T}\left(x_{0}\right)\right)-h\left(x_{0}\right)\right|\right] .
\end{aligned}
$$

Now the relation (14) with $\gamma=1$ leads to $\mathbb{E}\left[\left|h\left(F_{T}\left(x_{0}\right)\right)-h\left(x_{0}\right)\right|\right] \leq 2 A \cdot \mu \cdot T^{a-m}$. Then,

$$
\mathbb{E}\left[\left|Y_{T, 0}\right|\right] \leq 4 A \cdot \mu \cdot T^{a-m} .
$$

By the same arguments,

$$
\begin{aligned}
\mathbb{E}\left[\left(E_{0}^{(T)}\right)^{2}\right] & \leq 4 \mathbb{E}\left[\left(h\left(F_{T}\left(x_{0}\right)\right)-h\left(x_{0}\right)\right)^{2}\right] \\
& \leq 16 A^{2} \cdot \mu \cdot T^{2 a-m} \quad(\text { relation (14) with } \gamma=2) .
\end{aligned}
$$

b) Analogously, relation (14) with Hölder inequality yields

$$
\begin{aligned}
\left|\operatorname{Cov}\left(Y_{0}^{(T)}, E_{i}^{(T)}\right)\right| & \leq\left\|Y_{0}^{(T)}\right\|_{m / a} \cdot\left(\mathbb{E}\left[\left|E_{0}^{(T)}\right|^{\frac{m}{m-a}}\right]\right)^{\frac{m-a}{m}} \quad \text { (Hölder inequality) } \\
& \leq 2\left\|h\left(F_{T}\left(x_{0}\right)\right)\right\|_{m / a} \cdot 2\left(\mathbb{E}\left(\left|h\left(x_{0}\right)-h\left(F_{T}\left(x_{0}\right)\right)\right|^{\frac{m}{m-a}}\right)\right)^{\frac{m-a}{m}} \\
& \leq 2 A \cdot\left\|\left|x_{0}\right|^{a} \vee 1\right\|_{m / a} \cdot 2 A \cdot \mu^{\frac{m-a}{m}} \cdot\left(T^{-m\left(1-\frac{a}{m-a}\right)}\right)^{\frac{m-a}{m}} \quad(\text { assumptions on } h) \\
& \leq c \cdot A^{2} \cdot T^{2 a-m} .
\end{aligned}
$$

c) Let $h^{(T)}(u)=h\left(F_{T}(u)\right)-\mathbb{E}\left[h\left(F_{T}\left(x_{0}\right)\right)\right]$ for $u \in \mathbb{R}^{d}$. From assumptions on $h$, it can be shown that $\left\|h^{(T)}\right\|_{\infty} \leq 2 A \cdot T^{a}$ and $\operatorname{Lip} h^{(T)} \leq 2 A \cdot T^{a-1}$. Then the weak dependence inequality (3) implies:

$$
\begin{aligned}
\left|\operatorname{Cov}\left(Y_{0}^{(T)}, Y_{i}^{(T)}\right)\right| & \leq\left|\operatorname{Cov}\left(h^{(T)}\left(x_{0}\right), h^{(T)}\left(x_{i}\right)\right)\right| \\
& \leq 8 A^{2} \cdot T^{2 a-1} \cdot \eta_{i} .
\end{aligned}
$$

LEMMA 2. Let $h$ and $\left(x_{n}\right)_{n \in \mathbb{Z}}$ satisfy respectively assumptions $H$ and $A$, with $m>2 a$ and

$$
\alpha>\frac{m-1}{m-2 a} \quad \text { which implies } \sum_{i=1}^{\infty} \eta_{i}^{\frac{m-2 a}{m-1}}<\infty .
$$

Then:

a) The series $\left.\sigma^{2}=\sum_{i=-\infty}^{\infty} \operatorname{Cov}\left(h\left(x_{0}\right)\right), h\left(x_{i}\right)\right)=\sum_{i=-\infty}^{\infty} \operatorname{Cov}\left(Y_{0}, Y_{i}\right)$ converges; 
b) With $\sigma_{p}^{2}=\operatorname{Var}\left(\sum_{i=1}^{p} Y_{i}\right)$, there is a constant $c>0$ such that

$$
\left|\sigma^{2}-\frac{\sigma_{p}^{2}}{p}\right| \leq c \cdot\left(\frac{\log p}{p}+p^{\left(1-\frac{\alpha(m-2 a)}{m-1}\right)}\right) .
$$

Proof of Lemma 2. a) With $T_{i}>0$ for $i \in \mathbb{Z}$, we write

$$
\operatorname{Cov}\left(Y_{0}, Y_{i}\right)=\operatorname{Cov}\left(E_{0}^{\left(T_{i}\right)}, E_{i}^{\left(T_{i}\right)}\right)+\operatorname{Cov}\left(Y_{0}^{\left(T_{i}\right)}, E_{i}^{\left(T_{i}\right)}\right)+\operatorname{Cov}\left(Y_{i}^{\left(T_{i}\right)}, E_{0}^{\left(T_{i}\right)}\right)+\operatorname{Cov}\left(Y_{0}^{\left(T_{i}\right)}, Y_{i}^{\left(T_{i}\right)}\right) .
$$

From the previous lemma, $\left|\operatorname{Cov}\left(Y_{0}, Y_{i}\right)\right| \leq c \cdot A^{2} \cdot\left(T_{i}^{2 a-m}+T_{i}^{2 a-1} \cdot \eta_{|i|}\right)$. Now, set $T_{i}^{2 a-m}=T_{i}^{2 a-1} \cdot \eta_{|i|}$, then $T_{i}=\eta_{|i|}^{-\frac{1}{m-1}}>0$ and

$$
\left|\operatorname{Cov}\left(Y_{0}, Y_{i}\right)\right| \leq c \cdot \eta_{i}^{\frac{m-2 a}{m-1}}
$$

As a consequence, $\sum_{i=-\infty}^{\infty}\left|\operatorname{Cov}\left(Y_{0}, Y_{i}\right)\right| \leq c \cdot \sum_{i=-\infty}^{\infty} \eta_{i}^{\frac{m-2 a}{m-1}}$ and $\sigma^{2}$ exists thanks to the assumption (15).

b) Decompose $\sigma^{2}-\frac{\sigma_{p}^{2}}{p}=D_{1}+D_{2}$ with $D_{1}=\sum_{|i| \geq p} \operatorname{Cov}\left(Y_{0}, Y_{i}\right)$ and $D_{2}=\frac{1}{p} \sum_{|i|<p}|i| \cdot \operatorname{Cov}\left(Y_{0}, Y_{i}\right)$. From assumption (15), we conclude as above with inequality (17), because:

$-\left|D_{1}\right| \leq c \cdot \sum_{i \geq p} \eta_{i}^{\frac{m-2 a}{m-1}} \leq c \cdot p^{\left(1-\frac{\alpha(m-2 a)}{m-1}\right)}$, and

$-\left|D_{2}\right| \leq \frac{c}{p} \cdot \sum_{|i|<p}|i| \cdot \eta_{|i|}^{\frac{m-2 a}{m-1}}$. Now, $\left\{\begin{array}{l}\text { if } \alpha \geq 2 \cdot \frac{m-1}{m-2 a}, \text { then }\left|D_{2}\right| \leq c \cdot \frac{\log p}{p} \\ \text { if } \frac{m-1}{m-2 a}<\alpha<2 \cdot \frac{m-1}{m-2 a}, \text { then }\left|D_{2}\right| \leq c \cdot p^{\left(1-\frac{\alpha(m-2 a)}{m-1}\right)}\end{array}\right.$.

LEMMA 3. Let $h$ and $\left(x_{n}\right)_{n \in \mathbb{Z}}$ satisfy respectively assumptions $H$ and $A$, with $m>2 a$. For $p \in \mathbb{N}^{*}$, define: $W_{p}=\sum_{i=1}^{p} Y_{i}$. Then, if $\alpha>3$, for all $0<\delta<\frac{m-2 a}{a}$, there exists a constant $c>0$ such that:

$$
\mathbb{E}\left|W_{p}\right|^{2+\delta} \leq c \cdot p^{r} \quad \text { with } \quad \frac{2+\delta}{2} \leq r=2+\delta-\frac{m-2 a-a \cdot \delta}{m-1}<2+\delta .
$$

Proof of Lemma 3. Let $\Delta=2+\delta$ and $m=a(2+\zeta)$. With inequality (14) and $W_{p}^{(T)}=\sum_{i=1}^{p} Y_{i}^{(T)}$, we obtain:

$$
\left\|W_{p}\right\|_{\Delta} \leq\left\|W_{p}^{(T)}\right\|_{\Delta}+p\left\|Y_{0}-Y_{0}^{(T)}\right\|_{\Delta} \leq\left\|W_{p}^{(T)}\right\|_{\Delta}+c \cdot p \cdot T^{a-\frac{m}{\Delta}} .
$$

The Hölder inequality provides:

$$
\mathbb{E}\left|W_{p}^{(T)}\right|^{\Delta} \leq\left(\mathbb{E}\left|W_{p}^{(T)}\right|^{2}\right)^{1-\delta / 2}\left(\mathbb{E}\left|W_{p}^{(T)}\right|^{4}\right)^{\delta / 2}
$$

Now from c) of Lemma 1 , we obtain $\mathbb{E}\left|W_{p}^{(T)}\right|^{2} \leq c \cdot p \cdot T^{2 a-1} \sum_{i=0}^{\infty} \eta_{i}$. Setting

$$
C_{r, T}=\max _{u=1,2,3} \sup _{s_{u+1}-s_{u}=r}\left|\operatorname{Cov}\left(\prod_{i=1}^{u} Y_{s_{i}}^{(T)}, \prod_{i=u+1}^{4} Y_{s_{i}}^{(T)}\right)\right|
$$


where this supremum is set over $s_{1} \leq s_{2} \leq s_{3} \leq s_{4}$, we obtain as in (??),

$$
\mathbb{E}\left|W_{p}^{(T)}\right|^{4} \leq c\left(p \sum_{k=0}^{p-1}(k+1)^{2} C_{k, T}+\left(p \cdot T^{2 a-1} \sum_{i=0}^{\infty} \eta_{i}\right)^{2}\right) .
$$

We quote that $C_{k, T} \leq T^{4 a-1} \eta_{k}$ to derive

$$
\mathbb{E}\left|W_{p}^{(T)}\right|^{4} \leq c\left(p \cdot T^{4 a-1}+\left(p \cdot T^{2 a-1}\right)^{2}\right) .
$$

Thus, from previous inequalities and with $m=(2+\zeta) a$,

$$
\begin{aligned}
\mathbb{E}\left|W_{p}\right|^{\Delta} & \leq c\left(p^{\Delta} \cdot T^{a \Delta-m}+\left(p \cdot T^{2 a-1}\right)^{1-\delta / 2} \times\left(p \cdot T^{4 a-1}+p^{2} \cdot T^{4 a-2}\right)^{\delta / 2}\right) \\
& \leq c\left(p^{\Delta} T^{a(\delta-\zeta)}+\left(p \cdot T^{2 a-1}\right)^{\Delta / 2}+p \cdot T^{a \Delta-1}\right) .
\end{aligned}
$$

We now minimize this last inequality in $p$ by setting $T=p^{b}$ with $b>0$. With the condition $\delta<a \zeta$, we first show that it is necessary to have $b<1$ and the optimal $b$ is obtained by balancing of $p^{\Delta} T^{a(\delta-\zeta)}$ and $p \cdot T^{a \Delta-1}$. This value of $b$ is:

$$
b=\frac{1+\delta}{m-1},
$$

that satisfies $b<1$. We thus obtain $\mathbb{E}\left|W_{p}\right|^{\Delta} \leq c \cdot p^{2+\delta-\frac{a(\zeta-\delta)}{m-1}}$, that implies the result of the lemma.

3.0.0.1. Remark Notice that $r=2+\delta-\frac{m-2 a-a \cdot \delta}{m-1}>\frac{1}{2}$, contrarily to the classical MarcinkiewiczZygmund inequalities.

Proof of Theorem 1. We use a Bernstein blocks method for this proof. Consider three sequences of positive integers $p=(p(n))_{n \in \mathbb{N}}, q=(q(n))_{n \in \mathbb{N}}$ and $k=(k(n))_{n \in \mathbb{N}}$ such that:

$$
\begin{array}{ll}
\text { - } & \lim _{n \rightarrow \infty} \frac{p(n)}{n}=\lim _{n \rightarrow \infty} \frac{q(n)}{p(n)}=0 ; \\
\text { - } & \left.k(n)=\left[\frac{n}{p(n)+q(n)}\right] \text { (thus } \lim _{n \rightarrow \infty} k(n)=\infty\right) .
\end{array}
$$

These sequences are chosen as

$$
p(n)=\left[n^{\beta}\right], \quad q(n)=\left[n^{\gamma}\right], \quad \text { with } 0<\gamma<\beta<1,
$$

the exponents $\beta$ and $\gamma$ will be chosen below. We form the blocks $I_{1}, \ldots, I_{k}$ and define the random variables $U_{1}, \ldots, U_{k}$ such that:

$$
\begin{aligned}
I_{j} & =\{(j-1)(p(n)+q(n))+1, \ldots,(j-1)(p(n)+q(n))+p(n)\} \text { for } j=1, \ldots, k(n) ; \\
U_{j} & =\sum_{i \in I_{j}} Y_{i}, \text { for } j=1, \ldots, k(n) .
\end{aligned}
$$


Then expression (2) is decomposed as:

$$
\Delta_{n}=\sum_{\ell=1}^{3} \Delta_{\ell, n}
$$

where we set, for a standard Gaussian $N \sim \mathcal{N}(0,1)$,

$$
\begin{aligned}
& \Delta_{1, n}=\mathbb{E}\left(\phi\left(S_{n}\right)-\phi\left(\frac{1}{\sqrt{n}} \sum_{j=1}^{k} U_{j}\right)\right), \\
& \Delta_{2, n}=\mathbb{E}\left(\phi\left(\frac{1}{\sqrt{n}} \sum_{j=1}^{k} U_{j}\right)-\phi\left(N \sigma_{p} \sqrt{\frac{k}{n}}\right)\right), \\
& \Delta_{3, n}=\mathbb{E}\left(\phi\left(N \sigma_{p} \sqrt{\frac{k}{n}}\right)-\phi(\sigma N)\right) .
\end{aligned}
$$

Term $\Delta_{1, n}$. Using assumption (15) and a Taylor expansion up to order 2:

$$
\begin{aligned}
\left|\Delta_{1, n}\right| & \leq c \cdot\left(\frac{k(n) \cdot q(n)+p(n)}{n}\right) \frac{\left\|\phi^{\prime \prime}\right\|_{\infty}}{2} \sum_{i=0}^{\infty} \eta_{i}^{(m-2 a) /(m-1)} \\
& \leq c \cdot\left(n^{\beta-1}+n^{\gamma-\beta}\right) .
\end{aligned}
$$

Term $\Delta_{3, n}$. Now, Taylor formula implies:

$$
\left\{\begin{array}{l}
\phi\left(N \sigma_{p} \sqrt{\frac{k}{n}}\right)=\phi(0)+N \sigma_{p} \sqrt{\frac{k}{n}} \phi^{\prime}(0)+\frac{1}{2} N^{2} \sigma_{p}^{2} \frac{k}{n} \phi^{\prime \prime}\left(V_{1}\right) ; \\
\phi(N \sigma)=\phi(0)+N \sigma \phi^{\prime}(0)+\frac{1}{2} N^{2} \sigma^{2} \phi^{\prime \prime}\left(V_{2}\right),
\end{array}\right.
$$

with $V_{1}$ and $V_{2}$ two random variables. Then, with Lemma 2,

$$
\begin{aligned}
\left|\Delta_{3, n}\right| & \leq\left\|\phi^{\prime \prime}\right\|_{\infty} \cdot\left|\frac{k(n)}{n} \sigma_{p}^{2}-\sigma^{2}\right| \\
& \leq\left\|\phi^{\prime \prime}\right\|_{\infty} \cdot\left(\frac{p(n) \cdot k(n)}{n}\left|\sigma^{2}-\frac{1}{p(n)} \sigma_{p}^{2}\right|+\frac{n-p(n) \cdot k(n)}{n} \sigma^{2}\right) \\
& \leq c \cdot\left(\log (p(n)) \cdot p^{-1}(n)+p(n)^{1-\frac{\alpha(m-2 a)}{m-1}}+\frac{q(n)}{p(n)}\right) \\
\text { and therefore }\left|\Delta_{3, n}\right| & \leq c \cdot\left(n^{-\beta} \cdot \log n+n^{\beta-\frac{\alpha \cdot \beta \cdot(m-2 a)}{m-1}}+n^{\gamma-\beta}\right) .
\end{aligned}
$$

Term $\Delta_{2, n}$. Let $\left(N_{i}\right)_{1 \leq i \leq k(n)}$ be independent $\mathcal{N}\left(0, \sigma_{p}^{2}\right)$-Gaussian random variables, independent of the process $\left(x_{i}\right)_{i \in \mathbb{Z}}$ (such variables classically exist if the underlying probability space is rich enough). We define $\phi_{j}(t)=\mathbb{E}\left(\phi\left(\frac{1}{\sqrt{n}} t+\frac{1}{\sqrt{n}} \sum_{i=j+1}^{k(n)} N_{i}\right)\right)$ for $j=1, \ldots, k(n)$. In the sequel, 
for simplicity, empty sums are set equal to 0 . Then:

$$
\begin{aligned}
\Delta_{2, n} & =\mathbb{E}\left(\phi\left(\frac{1}{\sqrt{n}} \sum_{j=1}^{k(n)} U_{j}\right)-\phi\left(N \sigma_{p} \sqrt{\frac{k(n)}{n}}\right)\right) \\
& =\sum_{j=1}^{k(n)} \mathbb{E}\left(\phi\left(\frac{1}{\sqrt{n}} \sum_{i=1}^{j} U_{i}+\frac{1}{\sqrt{n}} \sum_{i=j+1}^{k(n)} N_{i}\right)-\phi\left(\frac{1}{\sqrt{n}} \sum_{i=1}^{j-1} U_{i}+\frac{1}{\sqrt{n}} \sum_{i=j}^{k(n)} N_{i}\right)\right) \\
& =\sum_{j=1}^{k(n)} \mathbb{E} \nu_{j, n},
\end{aligned}
$$

with $\nu_{j, n}=\phi_{j}\left(Z_{j}+U_{j}\right)-\phi_{j}\left(Z_{j}+N_{j}\right)$ and $Z_{j}=\sum_{i=1}^{j-1} U_{i}$.

Moreover, $\left\|\phi_{j}^{(\ell)}\right\|_{\infty} \leq n^{-\ell / 2}\left\|\phi^{(\ell)}\right\|_{\infty}$ for $\ell=0,1,2,3$. Making two distinct Taylor expansions (up to order 2 and 3 respectively) we obtain the two following expressions with some random variables $L_{j}$ for $j=1,2,3,4$ :

$$
\begin{aligned}
\left.\nu_{j, n}-\left[\phi_{j}^{\prime}\left(Z_{j}\right)\left(U_{j}-N_{j}\right)+\frac{1}{2} \phi_{j}^{\prime \prime}\left(Z_{j}\right)\left(U_{j}^{2}-N_{j}^{2}\right)\right)\right] & =\frac{1}{6}\left(\phi_{j}^{(3)}\left(L_{1}\right) U_{j}^{3}-\phi_{j}^{(3)}\left(L_{2}\right) N_{j}^{3}\right) \\
& =\frac{1}{2}\left[\left(\phi_{j}^{\prime \prime}\left(L_{3}\right)-\phi_{j}^{\prime \prime}\left(Z_{j}\right)\right) U_{j}^{2}\right. \\
& \left.-\left(\phi_{j}^{\prime \prime}\left(L_{4}\right)-\phi_{j}^{\prime \prime}\left(Z_{j}\right)\right) N_{j}^{2}\right] \\
\left.\mid \nu_{j, n}-\left[\phi_{j}^{\prime}\left(Z_{j}\right)\left(U_{j}-N_{j}\right)+\frac{1}{2} \phi_{j}^{\prime \prime}\left(Z_{j}\right)\left(U_{j}^{2}-N_{j}^{2}\right)\right)\right] \mid & \leq c\left(\frac{\left|U_{j}\right|^{2}}{n} \wedge \frac{\left|U_{j}\right|^{3}}{n^{3 / 2}}+\frac{\left|N_{j}\right|^{2}}{n} \wedge \frac{\left|N_{j}\right|^{3}}{n^{3 / 2}}\right) \\
& \leq \frac{c}{n^{1+\delta / 2}}\left(\left|U_{j}\right|^{2+\delta}+\left|N_{j}\right|^{2+\delta}\right)
\end{aligned}
$$

because the sequence $\left(N_{j}\right)_{j}$ is independent of the sequence $\left(x_{j}\right)_{j}$, and thus independent of the sequence $\left(U_{j}\right)_{j}$, and with the two relations $\mathbb{E} U_{j}^{2}=\sigma_{p}^{2}=\mathbb{E} N_{j}^{2}$ and $s^{2} \wedge s^{3} \leq s^{2+\delta}$ with $\delta \in[0,1]$ (that is valid for all $s \geq 0$ ). Now with the inequality $\mathbb{E}\left|N_{j}\right|^{2+\delta}=\left(\mathbb{E} U_{j}^{2}\right)^{1+\delta / 2} \mathbb{E}|\mathcal{N}(0,1)|^{2+\delta} \leq c \cdot \mathbb{E}\left|U_{j}\right|^{2+\delta}$ we derive

$$
\left|\mathbb{E} \nu_{j, n}\right| \leq\left|\operatorname{Cov}\left(\phi_{j}^{\prime}\left(Z_{j}\right), U_{j}\right)\right|+\frac{1}{2} \cdot\left|\operatorname{Cov}\left(\phi_{j}^{\prime \prime}\left(Z_{j}\right), U_{j}^{2}\right)\right|+\frac{c}{n^{1+\delta / 2}} \cdot \mathbb{E}\left|U_{j}\right|^{2+\delta} .
$$

Thus, using Lemma 3, with $C_{j}=\left|\operatorname{Cov}\left(\phi_{j}^{\prime}\left(Z_{j}\right), U_{j}\right)\right|$ and $C_{j}^{\prime}=\frac{1}{2}\left|\operatorname{Cov}\left(\phi_{j}^{\prime \prime}\left(Z_{j}\right), U_{j}^{2}\right)\right|$.

$$
\begin{aligned}
\left|\Delta_{2, n}\right| & \leq \sum_{j=1}^{k(n)}\left(C_{j}+C_{j}^{\prime}+c \cdot n^{-1-\delta / 2} p^{r}\right) \\
& \leq c \cdot n^{-\delta / 2} p^{r-1}+\sum_{j=1}^{k(n)}\left(C_{j}+C_{j}^{\prime}\right),
\end{aligned}
$$

Now, we can write the random variables $U_{j}, U_{j}^{2}, \phi_{j}^{\prime}\left(Z_{j}\right), \phi_{j}^{\prime \prime}\left(Z_{j}\right)$ as functions $G:\left(\mathbb{R}^{d}\right)^{u} \rightarrow \mathbb{R}$ of $x_{i_{1}}, \ldots, x_{i_{u}}$. The important characteristics of such $G$ are driven by the following respective orders: 
Random variable

$$
\begin{gathered}
U_{j}^{(T)} \\
\left(U_{j}^{(T)}\right)^{2} \\
\phi_{j}^{\prime}\left(Z_{j}\right) \\
\phi_{j}^{\prime \prime}\left(Z_{j}\right)
\end{gathered}
$$

Order $w$

$$
\begin{aligned}
& p(n) \\
& p(n) \\
& \leq n \\
& \leq n
\end{aligned}
$$

$\|G\|_{\infty}$

$\operatorname{Lip} G$

In order to use the weak dependence device for these two random variables $C_{j}$ and $C_{j}^{\prime}$, we have to use truncation $U_{j}^{(T)}$ obtained by replacing $Y_{i}^{\prime}$ 's by $Y_{i}^{(T)}$ and then,

$$
\left\{\begin{array}{l}
C_{j} \leq C_{j}^{(T)}+c \cdot\left\|\phi^{\prime}\right\|_{\infty} \cdot \frac{p(n)}{\sqrt{n}} \cdot \mathbb{E}\left|E_{0}^{(T)}\right| \text { with } C_{j}^{(T)}=\left|\operatorname{Cov}\left(\phi_{j}^{\prime}\left(Z_{j}\right), U_{j}^{(T)}\right)\right| ; \\
C_{j}^{\prime} \leq C_{j}^{\prime(T)}+c \cdot\left\|\phi^{\prime \prime}\right\|_{\infty} \cdot \frac{p^{2}(n)}{n} \cdot \mathbb{E}\left|Y_{0}^{2}-\left(Y_{0}^{(T)}\right)^{2}\right| \text { with } C_{j}^{(T)}=\frac{1}{2}\left|\operatorname{Cov}\left(\phi_{j}^{\prime \prime}\left(Z_{j}\right),\left(U_{j}^{(T)}\right)^{2}\right)\right| .
\end{array}\right.
$$

From the previous bounds, we obtain:

$$
\begin{aligned}
& C_{j}^{(T)} \leq c \cdot A^{2} \cdot\left(p(n) \cdot T^{2 a-1}+\left\|\phi^{\prime}\right\|_{\infty} \cdot p(n)^{2} \cdot T^{a-1} \cdot n^{-1 / 2}\right) \cdot \eta_{q(n)}, \\
& C_{j}^{\prime(T)} \leq c \cdot A^{3} \cdot\left(p(n)^{2} \cdot T^{3 a-1} \cdot n^{-1 / 2}+\left\|\phi^{\prime \prime}\right\|_{\infty} \cdot p(n)^{3} \cdot T^{2 a-1} \cdot n^{-1}\right) \cdot \eta_{q(n)},
\end{aligned}
$$

For this, one should mention that if $s \in \mathbb{N}^{*}$, the function $G_{T}^{(s)}$ defined on $\mathbb{R}^{d s}$ as $G_{T}^{(s)}\left(u_{1}, \ldots, u_{s}\right)=$ $\prod_{j=1}^{s}\left(h\left(F_{T}\left(u_{j}\right)\right)-\mathbb{E}\left[h\left(F_{T}\left(x_{0}\right)\right)\right]\right)$ satisfies $\left\|G_{T}^{(s)}\right\|_{\infty} \leq T^{s a}$ and $\operatorname{Lip} G_{T}^{(s)} \leq c \cdot A^{s} \cdot T^{s a-1}$.

Thus,

$$
\left\{\begin{array}{l}
C_{j} \leq c \cdot A^{3} \cdot\left(\frac{p(n) \cdot}{\sqrt{n}} T^{a-m}+\left(p(n) \cdot T^{2 a-1}+\frac{p^{2}(n)}{\sqrt{n}} T^{a-1}\right) \eta_{q(n)}\right) \\
C_{j}^{\prime} \leq c \cdot A^{3}\left(\frac{p^{2}(n)}{n} \cdot T^{2 a-m}+\left(\frac{p^{2}(n)}{\sqrt{n}} \cdot T^{3 a-1}+\frac{p^{3}(n)}{n} \cdot T^{2 a-1}\right) \cdot \eta_{q(n)}\right),
\end{array}\right.
$$

from relation (17), and because with inequalities (13),

$$
\mathbb{E}\left|Y_{0}^{2}-\left(Y_{0}^{(T)}\right)^{2}\right| \leq \mathbb{E}\left|E_{0}^{(T)}\right|^{2}+2 \mathbb{E}\left(\left|Y_{0}^{(T)}\right| \cdot\left|E_{0}^{(T)}\right|\right) \leq c \cdot T^{2 a-m} .
$$

Now, those bounds have to be minimized in $n$ by choosing $T$ as a function of $n$. We assume $\beta<1 / 2$ and hence $C_{j}$ and $C_{j}^{\prime}$ are minimized by selecting $T=n^{\frac{\alpha \gamma-1 / 2}{a+m-1}}$, that implies:

$$
\left\{\begin{array}{l}
C_{j} \leq c \cdot A^{3} \cdot n^{\beta-1 / 2-(\alpha \gamma-1 / 2)\left(\frac{m-a}{m+a-1}\right)} \\
C_{j}^{\prime} \leq c \cdot A^{3} \cdot n^{2 \beta-1-(\alpha \gamma-1 / 2)\left(\frac{m-2 a}{m+a-1}\right)}
\end{array}\right.
$$

under the conditions $\frac{1}{2 \alpha}<\gamma<\beta<\frac{1}{2}$. Finally, from (20), we obtain the following bound:

$$
\left|\Delta_{2, n}\right| \leq c \cdot A^{3} \cdot\left(n^{\beta(r-1)-\delta / 2}+n^{1 / 2-(\alpha \gamma-1 / 2)\left(\frac{m-a}{m+a-1}\right)}+n^{\beta-(\alpha \gamma-1 / 2)\left(\frac{m-2 a}{m+a-1}\right)}\right) .
$$


Therefore, inequalities (18), (19), (22) and condition $\frac{1}{2 \alpha}<\gamma<\beta<\frac{1}{2}$ provide:

$$
\left|\Delta_{n}\right| \leq c \cdot A^{3} \cdot n^{\max \left(p_{1}, p_{2}, p_{3}, p_{4}, p_{5}\right)} \quad \text { with }\left\{\begin{array}{l}
p_{1}=\beta\left(1-\alpha \cdot \frac{m-2 a}{m-1}\right) \\
p_{2}=\gamma-\beta \\
p_{3}=\beta\left(1+\delta-\left(\frac{m-2 a-a \delta}{m-1}\right)\right)-\delta / 2 \\
p_{4}=1 / 2-(\alpha \gamma-1 / 2)\left(\frac{m-a}{m+a-1}\right) \\
p_{5}=\beta-(\alpha \gamma-1 / 2)\left(\frac{m-2 a}{m+a-1}\right)
\end{array}\right.
$$

We have the possibility to make varying $\delta, \beta, \gamma$ (with certain conditions) for:

1. obtaining conditions on $\alpha$ and $m$, such that it exists $\delta, \beta$, $\gamma$ satisfying $\max \left(p_{1}, p_{2}, p_{3}, p_{4}, p_{5}\right)<0$;

2. minimizing $\max \left(p_{1}, p_{2}, p_{3}, p_{4}, p_{5}\right)$ with an optimal choice of $\delta, \beta, \gamma$ under the previous conditions.

To solve 1., the condition $p_{3}<0$ implies $\beta<\frac{m-2 a}{2(m-a)}$ with the optimal choice $\delta=m / a-2$. Moreover, condition $p_{4}<0$, implies $\gamma>\frac{1}{2 \alpha}\left(\frac{2 m-1}{m-a}\right)$. As a consequence, $\max \left(p_{1}, p_{2}, p_{3}, p_{4}, p_{5}\right)<$ 0 is satisfied when:

$$
\frac{1}{2 \alpha}\left(\frac{2 m-1}{m-a}\right)<\gamma<\beta<\frac{m-2 a}{2(m-a)} \quad \Longrightarrow \quad \alpha>\frac{2 m-1}{m-2 a} .
$$

To solve 2., fist we show that only coefficients $p_{2}, p_{3}$ and $p_{4}$ have to be considered for the minimization (under conditions (24), coefficients $p_{1}$ and $p_{5}$ are smaller than $p_{2}, p_{3}$ and $p_{4}$ ). Then, the optimal choice for $\gamma$ and $\delta$ is provided by the resolution of the system: $p_{2}=p_{3}$ and $p_{2}=p_{4}$, that implies to:

$$
\beta_{0}=\frac{m+2 a-1+\alpha(m-2 a)}{2(m+a-1+\alpha \cdot m)} \quad \text { and } \quad \gamma_{0}=\frac{3 m+2 a-2}{2(m+a-1+\alpha \cdot m)},
$$

and therefore, we obtain the optimal rate:

$$
\left|\Delta_{n}\right| \leq c \cdot A^{3} \cdot n^{-\lambda} \quad \text { with } \quad \lambda=\frac{\alpha(m-2 a)-2 m+1}{2(m+a-1+\alpha \cdot m)}
$$

\section{References}

Ango'nze, P. and Doukhan, P. Weak dependence: models and applications to econometrics. Econometric Theory, 20, 995-1045, 2004.

Bollerslev, T. Generalized autoregressive conditional heteroskedasticity. J. Econometrics, 31, 307-327, 1986.

Doukhan, P. Mixing: properties and examples. Lecture Notes in Statistics 85, Springer-Verlag, 1994.

Doukhan, P. Models inequalities and limit theorems for stationary sequences, in Theory and applications of long range dependence (Doukhan et alii ed.) Birkhäuser, 43-101, 2003.

Doukhan, P. and Lang, G. Rates in the empirical central limit theorem for stationary weakly dependent random fields. Stat. Inference Stoch. Process., 5, 199-228, 2002. 
Doukhan, P. and Louhichi, S. A new weak dependence condition and applications to moment inequalities. Stoch. Proc. Appl., 84, 313-342, 1999.

Doukhan, P., Madre, H. and Rosenbaum, M. ARCH-type bilinear weakly dependent models. Preprint available in http://www.crest.fr/pageperso/doukhan/bilinear.pdf, 2004.

Doukhan, P., Teyssiere, G. and Winant, P. Vector valued $\operatorname{ARCH}(\infty)$ processes. To appear in Lecture Note in Statistics, Special issu on time series, Bertail, P. , Doukhan, P. and Soulier, P. Editors, 2006.

Engle, R. F. Autoregressive conditional heteroscedasticity with estimates of the variance of United Kingdom inflation. Econometrica, 50, 987-1007, 1982.

Giraitis, L., Leipus, R. and Surgailis, D. Recent advances in ARCH modelling. In Long-Memory in Economics. G. Teyssière and A. Kirman editors, Springer Verlag (Forthcoming), 2005.

Giraitis, L. and Surgailis, D. A central limit theorem for quadratic forms in strongly dependent linear variables and its application to asymptotical normality of Whittle's estimate. Probab. Th. Rel. Fields, 86, 87-104, 1990.

Giraitis, L. and Surgailis, D. ARCH-type bilinear models with double long memory. Stoch. Proc. Appl., 100, 275-300, 2002.

Horvath, L. and Shao, Q.-M. Limit theorems for quadratic forms with applications to Whittle's estimate. Ann. Appl. Probab., 9, 146-187, 1999.

Leonov, V. P. and Shiryaev, A. N. On a method of semi-invariants. Theor. Probability Appl., 4, 319-329, 1959.

Rosenblatt, M. Stationary processes and random fields. Birkhäuser, Boston, 1985.

Rosenblatt, M. Gaussian and non-Gaussian linear time series and random fields. Springer Series in Statistics. Springer-Verlag, New York, 2000. 